\title{
Explaining how and explaining why: developmental and evolutionary explanations of dominance
}

\author{
ANYA PLUTYNSKI \\ Department of Philosophy, University of Utah, 260 S. Central Campus Dr. Rm. 341, Salt Lake City, \\ UT, 84112, USA (e-mail: plutynski@philosophy.utah.edu; phone: +1-801-581-7424; fax: +1-801- \\ $585-5195)$
}

Received 3 August 2005; accepted in revised form 19 September 2006

Key words: Dominance, Heterozygote, Homozygote, Wright, Fisher, Modifier genes, Genetic networks, Non-linear developmental pathways, Proximate v. ultimate causation

\begin{abstract}
There have been two different schools of thought on the evolution of dominance. On the one hand, followers of Wright [Wright S. 1929. Am. Nat. 63: 274-279, Evolution: Selected Papers by Sewall Wright, University of Chicago Press, Chicago; 1934. Am. Nat. 68: 25-53, Evolution: Selected Papers by Sewall Wright, University of Chicago Press, Chicago; Haldane J.B.S. 1930. Am. Nat. 64: 87-90; 1939. J. Genet. 37: 365-374; Kacser H. and Burns J.A. 1981. Genetics 97: 639-666] have defended the view that dominance is a product of non-linearities in gene expression. On the other hand, followers of Fisher [Fisher R.A. 1928a. Am. Nat. 62: 15-126; 1928b. Am. Nat. 62: 571574; Bürger R. 1983a. Math. Biosci. 67: 125-143; 1983b. J. Math. Biol. 16: 269-280; Wagner G. and Burger R. 1985. J. Theor. Biol. 113: 475-500; Mayo O. and Reinhard B. 1997. Biol. Rev. 72: 97$110]$ have argued that dominance evolved via selection on modifier genes. Some have called these "physiological" versus "selectionist," or more recently [Falk R. 2001. Biol. Philos. 16: 285-323], "functional," versus "structural" explanations of dominance. This paper argues, however, that one need not treat these explanations as exclusive. While one can disagree about the most likely evolutionary explanation of dominance, as Wright and Fisher did, offering a "physiological" or developmental explanation of dominance does not render dominance "epiphenomenal," nor show that evolutionary considerations are irrelevant to the maintenance of dominance, as some [Kacser H. and Burns J.A. 1981. Genetics 97: 639-666] have argued. Recent work [Gilchrist M.A. and Nijhout H.F. 2001. Genetics 159: 423-432] illustrates how biological explanation is a multi-level task, requiring both a "top-down" approach to understanding how a pattern of inheritance or trait might be maintained in populations, as well as "bottom-up" modeling of the dynamics of gene expression.
\end{abstract}

Dominance, as identified by Mendel (1865) in his garden peas, Pisum, occurs when, in the heterozygote condition (where there are two different alleles at a locus) a trait appears phenotypically exactly the same as one of the homozygotes (where there are the same alleles at a locus). For instance, in the pea, Mendel found that the factor that codes for the purple flowers is dominant over the hereditary factor for white, axial flower position is dominant over terminal position, yellow peas over green, and round over wrinkled. Dominance gives the characteristic 3:1 ratio of dominant to recessive types in the second generation. Subsequent to Mendel, however, biologists came to 
recognize varying degrees of dominance, such that the heterozygote is more or less like dominant type. Indeed, as soon as Mendel's work was rediscovered at turn of the century, many biologists were critical of Mendel's "all or none" concept of dominance (Correns 1900; Tschermak 1900). A more general definition of dominance is the non-additive allelic effects at a genetic locus on a phenotypic trait. This definition accommodates a wider variety of dominance phenomena, from "partial" to "complete" dominance, and treats dominance not as a static property of alleles, but an emergent phenotypic product of alleles, and often, multiple genes in interaction.

The phenomena of dominance have been long established. ${ }^{1}$ However, the explanation of dominance has been a long time coming. The debate over how best to explain dominance has a long history (see Falk 2001). Most recently, Gilchrist and Nijhout (2001) offered a new explanation of dominance, which seemed to render all prior accounts misconceived. Their paper, I will argue, raises a more general question: What is it to explain dominance? Do explanations in biology neatly divide into explanations of mechanism and explanations of function? I'll attempt to answer this question by examining one particular episode in the history of science, and then use that episode to illuminate the structure of arguments in the more recent literature. Arguably, the debate between Wright and Fisher over the proper explanation of dominance can be recast as a debate over whether developmental and evolutionary explanation are mutually exclusive, or supplementary.

\section{Historical overview}

First in 1922, and later, in 1928, Fisher put forth his explanation for the evolution of dominance. Fisher cited what he took to be three true generalizations about the patterns of dominance in transmission genetics. First, most mutations are deleterious and occurred at a finite, low rate. Second, most mutations are recessive to the wildtype. Third, the effect of such mutations was often to bring about the absence of some property found in the dominant type. He thus began his paper by citing the recent work of Morgan et al. (1925), where of 221 non-lethal mutations, 208 were classified as recessive and only thirteen as dominants. In addition, for traits due to multiple allelomorphs, Fisher noted that heterozygotes of non-wild-type genes tend to be intermediate. What he wanted to know was why this was the case, which, for him meant, how the condition of dominance in these traits could have evolved and been maintained in a population.

Prior to Fisher, Bateson and Punnett had offered the "Presence and Absence" hypothesis as an explanation for dominance. Their view was that the mutation was a loss, or an absence of whatever property lead to the presence of

\footnotetext{
${ }^{1}$ However, see below for a discussion in the recent philosophical literature on the "dissolution" of dominance.
} 
some trait in the wildtype. Or, mutation consisted in inactivation or dysfunction of the hereditary material. In modern terms, this explanation for dominance was that a single allele at a locus, the "dominant" allele, somehow "compensated" for loss, or mutation, at the other allele, either through providing an increase in the production of some enzyme, or through some other mechanism. Dominance, on this view, arises when a single allele is capable of the same effect as the wildtype. Incomplete dominance arises when a single gene cannot produce the same effects as a homologous pair. In the 1928 and later papers (1930b, 1931), Fisher argued that this explanation was unsatisfactory. He noted that there were often three or more alleles belonging to the same gene; so not all mutations could be an "absence." Second, he took the fact of reverse mutation to indicate that a mutation cannot be simply a loss.

Fisher's alternative hypothesis was as follows. First, he argued that a recurring deleterious mutation was likely to be held at a low frequency in a population. This is a straightforward result of the classic mutation-selection balance model, where the rate of new mutations is balanced by their elimination by selection (see Appendix). Starting with this simple mathematical model, Fisher showed how it might be possible for natural selection to modify heterozygotes such that they could exhibit the dominance effect.

Fisher's approach was a "top down" approach in the following sense; treat a phenotypic trait as the product of two alleles, and then determine under what conditions that trait can arise and be maintained over time, due to selection, mutation, etc.. For Fisher's purposes, I would argue, this is a harmless assumption. Fisher was well aware that many traits were due to genes at multiple loci; this, in fact, was the presupposition that permitted him to show the compatibility of Mendelism and the results of Biometry in 1918. However, treating a trait as the product of a single locus is useful for the purposes of modeling its dynamics over time. Of course, his approach made a number of additional assumptions. Fisher argued that when a mutation first arose it would most probably be in the heterozygote condition, and would most likely be deleterious to its carrier (a contentious assumption, but one that was not uncommon at the time). However, modifying genes, or perhaps, genes at adjoining loci that affect the same trait, might mitigate the deleterious effects of the recessive in the heterozygous condition. This was a version of the same thesis offered by East to explain gradual change in the striping pattern in hooded rats (East 1910). Fisher argued that natural selection would, over time, favor such modifiers, such that the heterozygotes come to resemble the homozygotes. That is, modifiers would be selected which should cause the heterozygote to more closely resemble the homozygote. In essence, Fisher's argument was that natural selection could satisfactorily explain the evolution of dominance.

Fisher's paper started a long discussion between Wright and Fisher, both in public and in private correspondence. Wright read Fisher's paper with great interest, since he had long been working on the inheritance of coat color in guinea pigs, and had long been interested in the physiology of gene expression. One of 
Wright's central research projects, both in his graduate work and beyond, at the USDA, was documenting the major factors in coat color. He found that most patterns were due to several genes in interaction. Although Wright was able to account for several of the patterns on a Mendelian system of crosses, much was left unexplained by single-factor Mendelian analysis. Wright wrote: "in each case a complex of the most varied causes underlies an apparently simple continuous series of variations" (Wright, in Provine, 1986b, p. 92). As a result of this research, Wright became convinced that each gene has multiple effects, and every gene will have different effects in different genetic backgrounds. In short, Wright was suspicious of single factor theories and top-down selective explanations, starting with idealized assumptions such as Fisher's.

Wright (1929) published a reply in the American Naturalist to Fisher's paper. First, Wright argued that the selective advantage of the modifier would be on the order of the mutation rate, too small by far for this to have an important effect in the evolution of dominance. Second, Wright cited evidence from Dobzhansky (1927) that genes have multiple effects, and argued that the modifier should also be expected to have multiple effects. In his words, modifiers would not be so "indifferent to selection on their own account." Wright thought that the modifiers might have significant effects on phenotype and fitness other than their effect on the "dominant" trait, and thus doubted that a selection coefficient on the order of the mutation rate alone would "control their fate," suggesting instead that drift might play a role. Further, Wright presented the then best available information on the physiology of gene action. He pointed out that genes act as catalysts, most likely bringing about the increase or decease in the production of enzymes. He suggested that "it seems that in the hypothesis that mutations are most frequently in the direction of inactivation and that for physiological reasons inactivation should generally behave as recessive, at least among factors with major effects, may be found the explanation of the prevalence of recessiveness among observed mutations"(Wright 1929, p. 69).

In short, Wright revisits the Presence-Absence theory, but it is far more sophisticated than its predecessor; inactivation, in his view, could occur in any number of ways, not simply via "loss of function" or gene product. In other words, Wright was gently changing the topic - in his view, the explanation of dominance was properly part of physiological genetics, the process of gene expression, and Fisher's explanation was implausible.

As a result of Wright's note, Fisher wrote to Wright and asked for clarification. His first impression was that Wright had misunderstood his mathematics: he asked whether Wright agreed that "a very slight selective effect acting for a correspondingly long time will be equivalent to a much greater effect acting for a proportionally shorter time." Wright's answer, it would turn out, was yes and no. Mathematically, Wright agreed that this could be the case. However, Wright thought that insofar as genes have multiple effects, the mere noise of such minor selection pressure would be quickly drowned out by much greater additional signals (perhaps random genetic drift), or other selective 
effects on the modifier. In other words, he invoked evidence in favor of an auxiliary hypothesis such that the deductive consequences of Fisher's mathematical argument would not follow. Wright had demonstrated that selection coefficients for modifiers would be on the order of mutation rate; such a small selection coefficient, he thought would cause mere fluctuations. Wright wrote to Fisher:

I assumed that the relative frequencies of any factor pair would be affected by many evolutionary pressures of varying order of magnitude. As the resultant of all such pressures there would be a certain equilibrium point but this point would practically be dependent (in this sense of approximate fixation of MM or $\mathrm{mm}$ [the modifier alleles]) on only the one or two most important pressures. Thus it seemed to me that selection and mutation pressures of a lower order would have virtually no effect, however long the period of time (Wright, in Provine, 1986b, p. 248).

Fisher responded, but he did not take Wright's criticism very seriously. In a rather abrupt paper to the American Naturalist, (1929) Fisher makes a few remarks on Wright's incorrect mathematics - he shows that the selective advantage of the modifier will be twice, not half, the mutation rate - but does not dispute Wright's main contention, that selection for modifiers will be on the order of mutation rate, and thus very minute. However, Fisher does not think that this is such a difficulty. He writes, "I do not in the least wish to dispute that the selective intensity will be proportional to, and generally of the order of, the mutation rate $k$, though the fact that the evolution of dominance by selection proceeds with increasing speed as dominance becomes more complete is an essential point stressed in my original note." In other words, Fisher thought that the difficulty was minor, and moreover, that as the selective advantage accrued by the modifier will increase with time, so too will the speed of fixation of the modifier allele, and thus the phenomenon of dominance. In other words, he invoked his own auxiliary hypothesis, thus accommodating the difficulties Wright brought to his attention, i.e., the auxiliary hypothesis that the advantageous effect of the modifier, and thus selective advantage, accrues with time.

It is clear that Fisher fully understood Wright's concern, but he did not think it compelling. Fisher makes little of Wright's suggestion that additional selective effects on the modifier will control its fate. His comment on Wright's note is worth citing in full, not only because it is one of the few places where Fisher comes close to suggesting what he expects will be the case in nature, but also because he makes some suggestive comments on parsimony, extrapolation and inference: 
He [Wright] suggests that the gene ratio of the modifying factors will either be held in equilibrium, so that a minute selective intensity will merely shift to a minute extent the position of equilibrium, and produce no progressive effect - as if the complex of gene ratios were a gel rather than a sol-or be irresistibly increased or decreased by selective intensities so powerful that a minute selective intensity can only delay or accelerate the extinction of the less favored gene ... much as a wind blowing along a railroad will not exert any affect in accumulating a rolling stock at the leeward terminal.

This is a well-directed criticism, but not a very strong one... My theory, in fact, is opposed, not by an obstinate fact of arithmetic, but by a rather original conjecture as to the inefficacy of minute selections...

There seems little probability of direct evidence upon so subtle a question. I have myself attached a good deal of importance, in other connections, to gene ratios held in stable equilibrium, though it never occurred to me that such a state was universal. Even if it were nearly so, the conditions on which the stability rest would be liable to change; stability may turn to instability, a transient state, admittedly, but one in which a minute, but steadily increasing selective intensity would be well fitted to tip the balance. As to the ratios having neutral stability, there is one reason for thinking that the factors suffering the feeblest selective action will at any one time be the most numerous. The fate of those powerfully selected is quickly settled... It is the idlers that make the crowd, and very slight attractions may determine their drift. On the whole, it seems that the most reasonable assumption, which we can make, on an obscure subject, is that the effect is approximately proportional to the cause (Fisher 1929, p. 556).

This is an illuminating passage, not only in illustrating how acerbic Fisher could be when crossed, but also in pointing out several important differences between Fisher and Wright. First, Fisher thinks that Wright's idea that the gene ratio is stable, or, primarily at equilibrium with minor fluctuations, is unlikely. In Fisher's view, the environment will be constantly changing, so even if the gene ratio is occasionally in equilibrium, this will quickly cease to be the case. A changing environment will cause changing fitness, i.e., selective values. This criticism of Wright would arise again in the context of Wright's adaptive landscape metaphor. In addition, Fisher argues that minute selective intensities can become stronger over time, since presumably the selective advantage of some trait will be increased when the trait comes to be expressed more fully. It further reveals that to Fisher, Wright's concern about multiple selective forces operating on single genes is inconsequential. It appears that he intended his talk about minute selective forces to be sufficient to reject Wright's point about multiple selective effects, or, the effects of other factors such as drift.

Fisher's remark about Wright thinking "as if the complex of gene ratios 'gel rather than a sol" is obscure. However, he may be suggesting here that he thinks that the genetic composition of a population is analogous to solution, 
where genes are relatively free of linkage, rather than like a 'gel', insofar as in every generation, gene combinations will be broken up by recombination. This was a key point for Fisher, and one that he thought answered the concern of Wright's as to genes in interaction. Fisher was well aware that genes had different effects in interaction. However, in Fisher's view, recombination would reverse any presumably "tightly integrated" adaptive combination. Fisher remarked on the effects of genes in combination:

It has, of course, long been known that genes that have a pronounced effect in the presence of some other gene may have none in its absence. Terms such as epistatic factors, complimentary factors, specific modifiers, etc., have only been introduced in recognition of particular cases of the general fact that the effect of any one genetic substitution depends on the gene complex, or genetic background, in which the substitution is made (Fisher 1931, p. 347).

Fisher's view was that this fact of genes in combination, in and of itself, should give one no reason to deny that a selective advantage of some gene will have an effect proportional to its cause. For, even though it is the combination of genes that affect the fitness of an organism, it is the gene itself that is either passed on or not. Thus, on average, in Fisher's view, a selective advantage, however slight, should lead to the fixation of some gene.

Further, in the above passage, Fisher briefly remarks on what he expects to be the genetic make-up of a population. It is interesting to note here that Fisher thinks most genes in a population will have a neutral or minor selective advantage, since it will simply take them longer either to fix or be eliminated from the population: "It is the idlers that make the crowd." In contrast, Fisher believes that genes of high or low selective advantage will be either quickly fixed or eliminated from the population.

Finally, Fisher thinks that Wright's objection rests upon an unsubstantiated claim that very small selection coefficients will be ineffective. In their present state of ignorance about gene expression, Fisher thinks it better to rely upon the conclusions that follow from his mathematics. Why not presume that small selective effects acting for a long time will be equivalent to large selective effects acting for a proportionally shorter time? In Fisher's view, without evidence to the contrary, why not assume that this consequence of his mathematics holds of the natural world? Over the long term, one might ignore interactions. It is interesting that this appeal to parsimony echoes the very same appeals made by both Castle and East in the context of modifier versus modified genes. (Castle 1906, 1914; East 1910) What counts as more or less parsimonious depends upon one's auxiliary theories about gene action and interaction. For Fisher, the view that small selection coefficients should have a small but eventual effect was common sense, particularly in light of his views on genetic interaction and selection. Why not presume that the effect is proportional to the cause? 
Wright thought he could show why not in this case. In his "Comment on Dr. Fisher's Reply" (1929), Wright took the debate a step further by introducing his equation for the probability distribution of an allele, and introduced in elementary form his shifting balance theory, which provided a larger context for the debate. He explained:

The question at issue reduces then to whether there are genes so neutral in relation to all other evolutionary forces that selections which change their frequencies at rates of the order of mutation pressure are the most important forces acting on them, and whether such genes are sufficiently numerous to give a basis for such a common phenomena as dominance. (Wright, in Provine, 1986b, p. 73.)

Wright was "skeptical" that this could be so for reasons more complex than he had mentioned before. As a result of his own mathematical investigations into the statistical consequences of Mendelism, Wright had concluded, "I do not hold that even the most important selective action on a gene is necessarily the controlling factor"'(Wright, in Provine, 1986b, p. 73). In particular, in a population of limited size, drift will lead to either the elimination or fixation of some allele, irrespective of an allele's minor selective advantage or disadvantage. Wright gives his formula for the probability distribution of an allele at frequency $q$ :

$$
\mathrm{e}^{2 \mathrm{nsq}} q^{4 \mathrm{nv}-1}(1-q)^{4 \mathrm{nu}-1}
$$

In the equation, $\mathrm{s}$ measures the selection favoring an allele, $\mathrm{u}$ is the mutation rate, $\mathrm{v}$ is the rate of reverse mutation, and $\mathrm{n}$ is the population size. Note that the above equation does not incorporate dominance, interactive effects of genes, or migration. Wright explains that this equation will take different forms under different conditions, depending jointly on population size, selection coefficients and mutation rates.

The form of the curve... indicates whether selection (s), isolation effect $(1 /(2 n))$ or mutation, $(\mathrm{u}, \mathrm{v})$ is the factor which controls the fate of the gene and thus in a sense adjudicates between the principles of Darwin, Wagner and DeVries, respectively (Wright, in Provine, 1986, p. 75).

It is interesting here that Wright is explicit about how his simple theoretical model unifies the views of three thinkers on the three major factors at work in evolution. In essence, his formula shows how no one of these three factors is the controlling force in evolution, but each in its turn may or may not be significant, depending upon the magnitude of the others. In particular: 
Selection controls the situation if $s$ is larger than $1 / 2 n$ but is of little importance below this figure. In small inbred populations $(1 / 2 n$ is large) even vigorous selection is ineffective in keeping injurious factors from drifting to fixation... In the case of Fisher's modifiers of dominance with selection coefficients at best of the order of mutation rate, the latter must be greater than $1 / 2 n$ if the gene is not to drift back and forth in the course of geologic time from one state of approximate fixation to the other and practically as freely in the face of the selection pressure as with it (Wright, in Provine, 1986b, p. 75).

Here Wright fully explains why he has come to reject Fisher's theory, connecting his views back with his own theoretical work, and stating exactly which assumptions about population size he relies upon. His argument rests upon the view that (1) most populations are of limited size, and one can expect that (2) selection coefficients of Fisher's modifier will not be greater than $1 / 2 n$. Recall that mutation rates are on the order of $10^{-6}$. If selection coefficients will be, as Fisher and Wright agree, no more than twice the mutation rate, then we will have selection rates of approximately $2 \times 10^{-6}$. However, as Wright explains above, selection coefficients must be greater than $1 / 2 n$ to be effective. It follows that for Fisher's argument for the evolution of dominance to work, then, population sizes in nature must be on the order of a million.

Wright admits that it is difficult to estimate population sizes in nature. However, he suggests that most populations are restricted to small localities, and seasonal oscillations may reduce population size significantly. Further, Wright brings additional information to bear on the question. If, he says, as some have suggested, non-adaptive differences between local races and subspecies may be explained by isolation and drift, then we may suspect that the quality of being isolated and having reduced population size will be very common in nature. Wright concludes the paper by suggesting that the explanation for dominance might better be sought in physiology and the inactivation of genes.

In subsequent publications (1930a, 1931), Fisher seems relatively unaffected by Wright's reply. In the case of The Genetical Theory, it is possible that Fisher's book was already in press when Wright's short paper came out. However, there is good reason to expect that even if Fisher had opportunity to revise, (which he did for subsequent editions (Fisher 1958)), he would not have seen it as necessary. Fisher argued that population sizes were on the order of the entire species, since small isolated populations are likely to die out, and migration connects local populations and renders them genetically contiguous with one another. Moreover, and more importantly for our purposes, Fisher thought that explanations of dominance at the level of the physiology of gene action needed to be supplemented by evolutionary explanations. It seems that Fisher viewed explanations at the level of the population as completing incomplete explanations, which appealed to physiology. The physiological explanation in terms of presence and absence, or activation or deactivation of 
some biochemical function, was incapable of accounting for the maintenance of dominance in populations, in Fisher's view. Whereas in Wright's view, in addition to the implausibility of selection shaping modifier alleles, Fisher's idealizing assumptions about the genetic bases of dominance rendered his evolutionary explanation inapplicable. Of course, it is possible that both explanations are necessary and complement, rather than contradict, one another. However, it seems that Fisher and Wright disagreed not only about the plausibility of the selection story Fisher was proposing, but also about the form of explanation.

Evidence that Wright's objections did have a minor effect on Fisher may be found in various passages of the 1931 "Evolution of Dominance" paper, which appeared in the Biological Review. First, the paper contains a great many more concrete examples of dominance in nature and in domestic species that Fisher uses to support his case. Moreover, he goes on to extend modifier theory and suggest that it may explain stable polymorphisms as well as dominance. Many of Fisher's remarks are along the lines of his reply to Wright in the earlier paper (1929). First, he remarks on how the efficacy of minute selective actions has been questioned (without stating by whom) and remarks that "since it is manifestly impossible to prove experimentally that a selective intensity of one ten-thousandth of another will really produce the same effect in ten thousand times that time, it will be better... to follow out the quantitative consequences of the theory." I.e., to assume that he is correct that effect is proportional to cause, and ultimately, that selection on modifiers is the preferred explanation. Second, Fisher again raises the point that one should expect the selective advantage of the modifier to increase: "We have seen that as the viability improves, the intensity of selection is greatly accelerated" (Fisher 1931, p. 352). It is the concluding statement of his 1931 paper that is most telling:

An interesting feature of the whole subject is that in nearly every case we are concerned with a minor or secondary bi-product of selective action. Anyone who accepts the view, which was propounded by some earlier geneticists, that selective agencies have been ineffective or unimportant in the morphological evolution of living forms, must of necessity, irrespective of the evidence, reject the view that it has been influential in the present group of cases. When, however, the matter is viewed, not with dogmatic partisanship, but in relation to the calculable magnitudes of the selective agencies at work, ... it is clear, as we have seen, even with the extremely minute selections favoring recessives in the mutants of Drosophila, that they are quantitatively of a magnitude sufficient to have produced the effects ascribed to them, provided their action has not been obstructed or opposed by some unknown and hypothetical cause (Fisher 1931, p. 367).

In the above passage Fisher seems to place those opposed to his views on the evolution of dominance in the category of those "earlier geneticists" who were 
skeptical of the power of selection. In other words, Fisher had placed Wright in the category of critics of Darwinism who did not view natural selection to be sufficient to explain morphological evolution. Moreover, his closing remark about "unknown and hypothetical" causes may be directed at Wright's earlier suggestion that modifiers may well be subject to other forces in addition to those suggested by Fisher (selective or drift). It seems that Wright's suggestion about population size restricting the effectiveness of very small selective effects only rendered Fisher more adamant. For Fisher, who saw much of his work as an attempt to vindicate Darwinism against his critics (see, e.g., the first chapter of 1930), any argument to the effect that selection would be ineffective was a challenge not only to any particular hypothesis, but to Darwinism as a whole.

The debate between Fisher and Wright over the evolution of dominance can be understood as a debate about the appropriate model of explanation. Fisher wished to give a top-down evolutionary explanation for dominance; or, his question was: "How could a pattern of dominance inheritance evolve and be maintained for any organism, for any trait?" He wanted a law-like understanding of this phenomenon. His answer involved an appeal to what was then known (and supposed) about the phenomena of dominance and mutation, as well as appeal to equilibrium and deterministic selection models. He argued that a recurring deleterious mutation was likely to be held at low frequency in a population. This follows from the mutation-selection balance model. Fisher suggested that it might be possible for natural selection to modify heterozygotes such that they could exhibit the dominance effect. He knew that when a mutation first arose it would be in the heterozygote condition, and would most likely be deleterious to its carrier. However, modifying genes at another locus might mitigate the deleterious effects of the recessive in the heterozygous condition. Natural selection could over time favor such modifiers, such that the heterozygotes come to resemble the homozygotes. That is, modifiers would be selected, which cause the heterozygote to resemble more closely the homozygote.

Wright disagreed with Fisher's evolutionary explanation, as well as Fisher's approach to the problem of explaining dominance. In other words, his disagreement was in part an objection to Fisher's actual explanation, but also to his top-down approach, which ignored the physiological mechanisms that could give rise to dominance patterns of inheritance. For Wright, explaining dominance required both evolutionary and physiological considerations.

Wright's objection to Fisher's evolutionary explanation was part and parcel of his larger disagreement with Fisher over the significance of selection in evolution. As Wright notes in the final pages of his (1934) "Physiological and Evolutionary Theories of Dominance":

My interest in [Fisher's] theory of dominance was based in part on the fact that I had reached a very different conception of evolution (1931) and one to which his theory of dominance seemed fatal if correct. As I saw it, selection could exercise only a loose control over the momentary 
evolutionary trend of populations. A large part of the differentiation of local races and even of species was held to be due to the cumulative effects of accidents of sampling in populations and limited size. Adaptive advance was attributed more to intergroup than intragroup selection (Wright, 1934, p. 200).

In Wright's view, selection simply did not play such a pervasive role in shaping the genetic constitution of populations as it did in Fisher's view. Thus, Fisher's suggestion that the loci yielding dominance patterns of inheritance could be shaped by selection struck him as implausible. In Wright's view, such intragroup evolution was most likely due in large part to sampling (drift) in populations of limited size. In other words, gene complexes exhibiting patterns of dominance were most likely products of accidental sampling, occasionally modified by selection, or, "selection could exercise only a loose control."

Moreover, Wright thought that dominance phenomena were most likely multiply realizable - there were a variety of genetic interactions that could plausibly yield dominance patterns of inheritance (1934). Wright's offered a "physiological" theory of dominance. Dominance, in Wright's view, "has to do with the physiology of the organism and has nothing to do with the mechanism of inheritance, i.e., with heredity in the narrow sense" (Wright 1934, p. 173) Genes act as catalysts, most likely bringing about the increase or decrease in the production of enzymes. Since mutations (he thought) are most frequently in the direction of inactivation, and since inactivation should generally behave as recessive, we should, and we do, find most mutation to be recessive (Wright 1934). Dominance is a product of enzymatic activity, which, he thought, might exhibit a specific dynamic relationship between variation in product $(\mathrm{Y})$ and variation in catalyst $(\mathrm{X})$. Wright suggested that the relationship between the two should be hyperbolic, and asymptotic at its upper limit. Thus, doubling the quantity of $\mathrm{X}$ will less than double the amount of the product. Dominance phenomena thus exhibited a sort of "law of diminishing returns"; the amount of enzyme produced by some allele yields an asymptotic gene/product curve. So, degrees of dominance are simply byproducts of the dosage effects of this curve (Wright 1929, 1934).

It is important to note that Wright understood this model to be hypothetical, and moreover, he did not view it as necessarily excluding a role for evolutionary considerations in explaining dominance. Wright disagreed with Fisher's approach to dominance not because he thought evolutionary considerations were somehow excluded by physiological explanations, as subsequent authors have argued (Kacser and Burns 1981). ${ }^{2}$ Rather, Wright simply disagreed with Fisher's favored evolutionary explanation. Wright argued that, as there are a variety of gene complexes that might give rise to

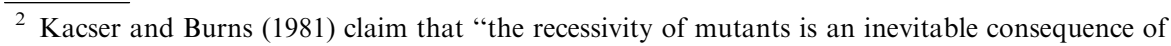
kinetic properties of enzyme related pathways and that no other explanation is required" (Kascer and Burns 1981, p. 640).
} 
patterns of dominance inheritance in some trait, the evolutionary mechanism by which this particular gene complex arose or might be maintained might be different in each case. Wright explains:

It must be remembered that whatever the evolutionary mechanism by which a particular gene complex has been reached, the state of dominance of all the genes in the complex must always have a completely physiological explanation (Wright, 1934, p. 179).

Wright's claim is not that evolutionary considerations are irrelevant, but that one must realize that the appearance of dominance will have a physiological explanation as well. Wright is not saying that dominating traits cannot be subject to evolution: "I have no objection to an evolutionary process by which the dominance of wildtype over mutations may be increased provided the pressure toward selection is sufficient to be effective" (Wright 1934, p. 199). In other words, the point of difference between Wright and Fisher is not over the object of explanation per se, but how to explain it.

This view of Wright and Fisher differs slightly from that found in the literature so far. Falk (2001), for instance, has argued that Wright had a "functional" view of dominance, which he contrasts with Fisher's "structuralist" view, arguing that Fisher saw dominance as a property of the genotype, whereas Wright viewed dominance as a property of the phenotype. It's clear that Fisher made idealizing assumptions about the genetic bases of dominance, and Wright was correct to criticize these assumptions. However, from the above examination of their views, it seems that both Wright and Fisher viewed dominance as a property of phenotypes - a pattern of inheritance in some trait, underpinned by alleles, as well as genes, in interaction. Where they disagreed is in what a comprehensive explanation of dominance should look like. Wright disputed Fisher's "top-down" approach because he thought that (a) it idealized, at least, and misconstrued, at worst, the genetic bases of dominance, (b) selection for rare modifier genes in any case would not be strong enough, and (c) the fact that dominance was multiply realized meant that the role of evolution in maintaining or leading to dominant traits might be different in each case. Thus, for Wright, explaining dominance was a multi-level affair, requiring both physiological and evolutionary considerations. For Fisher, the complications of genetic bases might be ignored or idealized away.

\section{The "dissolution of dominance"?}

A number of recent papers in the philosophical literature have surveyed the history of dominance (Falk 2001), suggesting that at in at least some branches of biology (e.g., molecular genetics), dominance has disappeared from the lexicon, and questioning whether the concept is loaded with so many unwarranted assumptions that it ought to be abandoned (Allchin 2005, 2002). Allchin has argued, for instance, that: 
The current notion of dominance is steeped in a culturally and scientifically inappropriate metaphor, leads to inconsistencies or awkward contingencies, and has been misleading historically (Allchin, 2005, p. 440).

This seems a significant enough challenge to the whole project of explaining dominance that it is worth addressing these points at least briefly before turning to an analysis of what the Fisher-Wright debate might illuminate about biological explanation.

First, Allchin makes a persuasive case that the metaphor of "dominance" has led to unfortunate "assumptions and default conceptualizations," among students, the public, and also in the scientific context. Students (and the public) often falsely assume that a dominant trait is somehow "stronger," that is it more likely to be inherited, more fit, or prevalent (Allchin 2005, p. 429). Scientists too, are not immune. For instance, as Paul (1995) points out, many false assumptions about dominance infected the early eugenics movement, leading to sterilization of thousands of criminals and the insane. There is no doubt, as Allchin says, that "The language [of dominance] engenders false beliefs" (Allchin 2005, p. 429), which in turn, have lead to morally objectionable, as well as scientifically unsound practices.

In addition, Allchin and Falk are correct to point out that dominance is difficult to characterize. First, the term "dominance" is used to refer to different properties: phenotypic traits, or allelic variants are referred to as "dominant" in different scientific contexts. Second, there is not a single mechanism underpinning the phenomena of dominance, the extent of dominance in a phenotype may depend upon a suite of other alleles at loci other than the major "gene for" such a trait (there is more often than not multiple loci effecting some trait), and, at different levels of organization there are different degrees or kinds of dominance at work. Pleiotropy also complicates dominance attribution. Allchin is absolutely correct to draw attention to these problematic aspects of dominance. However, he draws some rather strong conclusions on the basis of these observations. He suggests, for instance, that "The language of dominance might readily dissolve," and that dominance is "epiphenomenal," explaining that:

Dominance embodies the linear, billiard-ball model of causality, which emphasizes singular causes, thereby obscuring context and overstating effects... A more modest sense of causality focuses on causal factors, rather than complete causes (Allchin, 2005, p. 440).

There is reason to take issue with both of these suggestions. First, it's not clear that we should or can expect the language of dominance to be eliminated from scientific discourse. In addition to the problem that scientific terms have inertia, there is good reason to keep "dominance": it remains a useful heuristic. In medical genetics, for instance, it is extremely useful to investigate whether a 
trait follows a pattern of dominance inheritance, even if the simple Mendelian model surely simplifies the underlying genetic bases of these traits. Further, it is not clear exactly what Allchin means by the claim that dominance "embodies" a "linear billiard-ball model of causality." Historically, dominance is one of the few cases in the history of early genetics where geneticists acknowledged the combined effects of genes in producing phenotypes. Wright's physiological genetic models of genes in interaction, and, even Fisher and Ford's "modifier" genes are not, strictly speaking, dealing with "linear" one to one effects of single genes on traits. Insofar as dominance always depends on several alleles in interaction, or, as defined above, it consists in "non-additive" effects of alleles, it is an early example of biologists' recognition that the causal effects of alleles are not "linear," at least in the sense of non-additive. In part for these very reasons, Sarkar has argued (1998) that dominance has turned out to be one of those troublesome cases from transmission genetics that has resisted reduction to molecular genetics.

Allchin suggests that the "reification" of dominance is problematic. This is a much more controversial claim; and, addressing this in great detail might require a longer paper. Yet, it's interesting that Allchin discusses a variety of cases of dominance (sickle cell, Tay Sachs, etc.), admittedly in the service of illustrating how and why identifying dominance as a property of traits, or alleles, or both, is highly problematic. Allchin's argument for the claim that dominance is epiphenomenal seems to be that while there are some cases of phenotypic traits that exhibit dominance patterns of inheritance, most cases of inheritance depart from this model, and moreover, even for those cases that do follow the model, the mechanisms differ. In his words:

Dominance as a type has no single general mechanism... In terms of reduction, the relationship is many-many. Dominance cannot serve as a shorthand for a consistent causal mechanism (Allchin, 2005, p. 440).

Allchin is correct that the notion of dominance can mislead in any number of ways, and that dominance is subserved by a variety of biological mechanisms; it is a "many-many" relation. However, it does not follow that dominance is epiphenomenal. Much of what Allchin says of dominance might also be said of any number of biological concepts. To take a (rather contentious) example, consider "fitness." Like "dominance," "fitness" is a "many-many" relation. Many mechanisms subvene fitness, and fitness may alternately be attributed to traits or alleles, or genotypes, in various modeling contexts. Also like dominance, pleiotropy complicates attributions of fitness, and fitness always depends upon many genes and alleles in interaction. However, we should not draw the conclusion that the notion of fitness should be "dissolved," nor, that fitness is epiphenomenal (Shapiro and Sober 2006). It seems clear, at least for now, anyway, that fitness is a useful explanatory concept in biology, and so too, is "dominance." At the very least, understanding how and why biologists 
have disagreed about the explanations for dominance can shed light on the nature of evolutionary explanation.

\section{Conclusions}

Recently, Nijhout and Paulson (1997) and Gilchrist and Nijhout (2001) have used genetic network theory to show that Fisher and Wright are both, partially, correct. More precisely, their conclusions are consistent both with the view that selection can lead to changes in dominance by changes in related loci, and that dominance results from the non-linearities underlying physiology.

In sum, their work demonstrates that explaining dominance requires understanding at different levels and time scales - not only functional explanations, but also, explanations of the kinetic properties of enzymatic pathways, and genetic regulatory networks underpinning development. In their (2001) piece, Gilchrist and Nijhout show that dominance can emerge out of non-linear relationships among alleles controlling the developmental process. In their words, "Although the dominance we observe... is the result of a physiological process, we also find that dominance can evolve by microevolutionary mechanisms and thus are able to reconcile the opposing views of Fisher and Wright on dominance" (Gilchrist and Nijhout, 2001, p. 423). In other words, there is a developmental story to tell about dominance; dominance is a product of gradient thresholds in embryological development. However, it is also possible to model the evolution of dominance from the "top down" using a mathematical model treating changes in dominance as products of modifying alleles. In other words, dominance can be investigated and explained both from the "bottom up" and the "top down." Using a mathematical model, Gilchrist and Nijhout arrive at the conclusion that "for any non-linear genotype-phenotype mapping, irrespective of the mechanism bringing it about, dominance will emerge." In other words, dominance is a byproduct of certain kinds of developmental systems. Nonetheless, developmental explanations do not exclude population level evolutionary explanations. Selection also is important to understand the process and evolution of dominance: dominance is "not a static property of a particular developmental or genetic mechanism, but is sensitive to allelic variation, and should, therefore, be subject to microevolutionary change" (Gilchrist and Nijhout, 2001, p. 424).

It seems clear that what it is to explain general patterns in biology, like dominance, is not easily resolved into Mayr's discrete categories of "proximate" and "ultimate" causation. Explaining such a complex phenomena as dominance requires that one draw upon knowledge from different levels of organization and different times scales. Physiological genetics are important, but so too are the dynamics of gene expression and the dynamics of selection on allelic variation. It was not simply the "developmental perspective" that allowed this insight, but an integration of developmental and evolutionary thinking. Moreover, construction of a general model, in this case, gave rise to surprising results. Dominance 
emerges out of complex, non-linear developmental processes. So, this is a nonreductive, non-linear causal explanation - one which requires integrating results from several different domains of biology, and, moreover, shows how neither Wright nor Fisher were, completely "right" in the end.

The lesson of this example is an interesting one for philosophers of science. There is, unfortunately, still a divide between philosophers who argue that molecular explanations will supplant evolutionary explanations, and those who see a role for both. This case shows how a comprehensive understanding of phenomena such as dominance requires both an understanding of the molecular and developmental mechanisms generating it, but also the construction of a general model, and an understanding of how selection can shape that system.

\section{Acknowledgements}

Thanks to Sahotra Sarkar, Ron Amundson, Steve Downes, and two anonymous reviewers for their generous feedback.

\section{Appendix}

Mutation Selection Balance (From Maynard Smith 1989)

Consider a large population of size $N$, where $a$ is the wildtype allele at frequency $q$, and $A$ is the mutant allele, at frequency $p$. So:

\begin{tabular}{llll}
\hline Genotypes & $a a$ & $A a$ & $A A$ \\
\hline Fitness & 1 & $1-h s$ & $1-s$ \\
Number of zygotes & $N q^{2}$ & $2 N p q$ & $N p^{2}$ \\
\hline
\end{tabular}

There are $2 N p q A a$ zygotes, of which a proportion $h s$ die, eliminating one $A$ gene. Further, there are $N p^{2} A A$ homozygotes, of which s die in each generation, eliminating two $A$ genes with each death. So, the number of $A$ genes lost by selection in each generation will be

$$
2 N_{p q h s}+2 N_{p 2 s}=2 N p s(q h+p)
$$

Further, in each new generation, new $A$ genes arise by mutation. Let the mutation rate be $\mathrm{u}$. Thus, since there are $2 N q$ a genes in each population, there will be $2 N q u$ new $A$ genes each generation. At equilibrium, (mutation-selection balance), the number of new mutations will equal the number eliminated. Or:

$$
2 N_{q u}=2 N_{p s}(q h+p)
$$


Or,

$$
q u=p s(q h+p)
$$

If $A$ is fully recessive to a, or $h=0$, then $q u=p^{2} s$, so, since $\mathrm{p}$ approximately $=1$

$$
p=\sqrt{ }(u / s)
$$

\section{References}

Allchin D. 2005. The dilemma of dominance. Biol. Philos. 20: 427-451.

Allchin D. 2002. Dissolving dominance. In: Parker (ed.), Mutating Concepts, Evolving Disciplines: Genetics, Medicine and Society, Kluwer, Dordrecht, pp. 43-62.

Bürger R. 1983a. Dynamics of the classical genetic model for the evolution of dominance. Math. Biosci. 67: 125-143.

Bürger R. 1983b. Nonlinear analysis of some models for the evolution of dominance. J. Math. Biol. 16: $269-280$.

Castle W.E. 1906. Yellow mice and gametic purity. Science 31: 275-281.

Castle W.E. 1914. Piebald rats and selection: an experimental test of the effectiveness of selection and of the theory of gametic purity in Mendelian crosses. Carnegie Inst. Publ. 195: 1-56.

Correns C. G. (1900) (1950). "Mendel's law concerning the behavior of progeny in varietal hybrids" (Eng. Trans.), Genetics 35(5:2): 33-41.

Dobzhansky T 1927. Studies on the manifold effects of certain genes in Drosophila Melanogaster. Z. Indukt. Abstamm. Vererbungfl. 43: 330-388.

East E.M. 1910. A Mendelian interpretation of variation that is apparently continuous. Am. Nat. 44: 65.

Falk R. 2001. The rise and fall of dominance. Biol. Philos. 16: 285-323.

Fisher R.A. 1922. On the dominance ratio. Proc. R. Soc. Edinburgh 42: 321-341.

Fisher R.A. 1928a. The possible modification of the response of the wild type to recurrent mutations. Am. Nat. 62: 15-126.

Fisher R.A. 1928b. Two further notes on the origin of dominance. Am. Nat. 62: 571-574.

Fisher R.A. 1929. The evolution of dominance: a reply to Professor Sewall Wright. Am. Nat. 63: 553-556.

Fisher R.A. 1930a. The genetical theory of natural selection. Oxford: Clarendon press. 2nd ed., 1958. New York: Dover.

Fisher R.A. 1930b. The distribution of gene ratios for rare mutations. Proc. R. Soc. Edinburgh 50: 205-220.

Fisher R.A. 1931. Evolution of dominance. Biol. Rev. 6: 345-368.

Gilchrist M.A. and Nijhout H.F 2001. Nonlinear developmental processes as sources of dominance. Genetics 159: 423-432.

Haldane J.B.S. 1930. A note on Fisher's theory of the origin of dominance and linkage. Am. Nat. 64: 87-90.

Haldane J.B.S. 1939. The theory of the evolution of dominance. J. Genet. 37: 365-374.

Kacser H. and Burns J.A. 1981. The molecular basis of dominance. Genetics 97: 639-666.

Maynard Smith J. 1989. Evolutionary Genetics. Oxford University Press, Oxford.

Mayo O. and Reinhard B. 1997. The evolution of dominance: a theory whose time has passed?. Biol. Rev. 72: 97-110.

Morgan T.H., Bridges C. and Sturtevant A. 1925. The Genetics of Drosophila. Martinus Nijhoff, The Hague.

Mendel, G. 1865. Experiments in Plant Hybridization. Proceedings of the Natural History Society of Bruenn, pp. 3-47. 
Nijhout H.F. and Paulsen S.M 1997. Developmental models and polygenic characters. Am. Nat. 149: 394-405.

Paul D. 1995. Controlling Human Heredity: 1865 to the Present. Humanity Books.

Provine W. 1986a. Sewall Wright and Evolutionary Biology. University of Chicago Press, Chicago. Provine W. 1986b. Evolution: Selected Papers by Sewall Wright. University of Chicago Press, Chicago.

Provine, W. 1992. The R.A. Fisher-Sewall Wright controversy. In: Sarkar (ed.), The Founders of Evolutionary Genetics, pp. 201-229.

Sarkar S. 1998. Genetics and Reductionism. Cambridge University Press, Cambridge.

Shapiro L. and Sober E. 2006. Epiphenomenalism - The Do's and The Don'ts. In: Wolters G. and Machamer P. (eds.), Studies in Causality: Historical and Contemporary, University of Pittsburgh Press, Pittsburgh.

Tschermark E. 1900 (1950). Concerning Artificial crossing in Pisum sativum." Genetics 35(5:2): 42 47.

Wagner G. and Bürger R 1985. On the evolution of dominance in modifiers. II A non-equilibrium approach to the evolution of genetic systems. J. Theor. Biol. 113: 475-500.

Wright S. 1923a. Mendelian Analysis of Pure Breeds of Livestock I: The Measurement of Inbreeding and relationship. J. Hered. 14: 339-348, In: Provine (ed.) 1986, Evolution: Selected Papers by Sewall Wright, University of Chicago Press, Chicago.

Wright S. 1923b. Mendelain Analysis of Pure Breeds of Livestock II: The Duchess Family of Shorthorns as Bread by Thomas Bates. J. Hered. 14. 405-422. In: Provine (ed.) 1986, Evolution: Selected Papers by Sewall Wright, University of Chicago Press, Chicago.

Wright S. 1925. The Shorthorns, J. Hered. 16(6): 205-215. In: Provine (ed.) 1986, Evolution: Selected Papers by Sewall Wright, University of Chicago Press, Chicago.

Wright S. 1929. Fisher's Theory of Dominance. Am. Nat. 63: 274-279. In: Provine (ed.) 1986, Evolution: Selected Papers by Sewall Wright, University of Chicago Press, Chicago.

Wright S. 1930. Review of the Genetical Theory of Natural Selection by R.A. Fisher. J. Hered. 21. 349-356. In: Provine (ed.) 1986, Evolution: Selected Papers by Sewall Wright, University of Chicago Press, Chicago.

Wright S. 1931. Evolution in Mendelian Populations. Genetics 16: 97-159. In: Provine (ed.) 1986, Evolution: Selected Papers by Sewall Wright, University of Chicago Press, Chicago.

Wright S. 1932. The Roles of Inbreeding, Crossbreeding and Selection in Evolution. Proceedings of the Sixth International Congress of Genetics 1: 356-366. In: Provine (ed.) 1986b, Evolution: Selected Papers by Sewall Wright, University of Chicago Press, Chicago.

Wright S. 1934. Physiological and Evolutionary Theories of Dominance. Am. Nat. 68: 25-53. In: Provine (ed.) 1986b, Evolution: Selected Papers by Sewall Wright, University of Chicago Press, Chicago.

Wright S. 1978. The Relation of Livestock Breeding to Theories of Evolution, J. Anim. Sci. 46: 1192-1200. In: Provine (ed.) 1986b, Evolution: Selected Papers by Sewall Wright, University of Chicago Press, Chicago. 